\title{
Avaliação de Plataforma Multimodal de Jogos Sérios para Auxílio a Terapia para Autistas
}

\author{
André B. Trindade ${ }^{1}$, Joice L. Jeronimo ${ }^{1}$, Marcelo da S. Hounsell ${ }^{2}$, Rafael G. Faust ${ }^{1}$ \\ ${ }^{1}$ Departamento de Engenharia Elétrica - Instituto Federal de Santa Catarina (IFSC) - \\ Joinville, SC - Brasil \\ ${ }^{2}$ Departamento de Ciência da Computação - Universidade do Estado de Santa Catarina \\ (UDESC) - Joinville, SC - Brasil \\ \{andre.bonetto, joice.jeronimo, rafael.faust\}@ifsc.edu.br, \\ marcelo.hounselleudesc.br
}

\begin{abstract}
This work describes the development of a platform capable of capturing heart rate through a non-invasive optical sensor built into a joystick. The platform focuses on autism spectrum disorder (ASD) whose interaction with digital media is yet to be fully understood. A serious game was developed to test the heart rate signal as a game parameter. A questionnaire was completed by 39 experts to evaluate the platform, the design of the joystick and the game. The experts assessed all 18 items above the center of the scale, except project costs. We can conclude that this type of multimodal platform can improve the possibilities of creating specific serious games for $A S D$.
\end{abstract}

Resumo. Este trabalho descreve o desenvolvimento de uma plataforma capaz de capturar a frequência cardíaca através de um sensor óptico não invasivo embutido em um joystick. A plataforma se concentra nos transtornos do espectro autista (TEA), cuja interação com mídias digitais ainda não foi totalmente compreendida. Um jogo sério foi desenvolvido para testar a frequência cardíaca como parâmetro de jogo. Um questionário foi respondido por 39 especialistas que avaliaram a plataforma, o design do joystick e o jogo. Todos os 18 itens foram avaliados acima do centro da escala, exceto os custos do projeto. Podemos concluir que este tipo de plataforma multimodal pode melhorar as possibilidades de criação de jogos sérios específicos para TEA.

\section{Introdução}

No Brasil, estima-se uma população de 2 milhões de pessoas com transtornos do espectro autista (TEA) [Oliveira 2016]. Atualmente, TEA não tem cura, contudo, estudos [Warren 2011] mostram que intervenções precoces podem melhorar o desenvolvimento de crianças autistas e estas são geralmente realizadas nas áreas deficientes mais comuns, como aquelas que trabalham o desempenho cognitivo da criança, habilidades de comunicação e comportamentos adaptativos.

Intervenções que utilizam recursos tecnológicos como computadores, tablets e outros dispositivos, vêm se mostrando eficientes e atrativas para o público autista [Galvão 2012]. Neste contexto, os jogos digitais sérios (JS) podem se tornar uma alternativa interessante para o TEA. JS são aplicações digitais desenvolvidas com a 
participação de especialistas, tendo a intenção original de combinar um propósito específico sério com o divertimento que um vídeo game pode proporcionar [Alvarez e Djaouti 2011]. O uso de JS para o estímulo a autistas é ainda uma área nova, onde a própria interação com o JS é um fator a ser melhor entendido. Como as intervenções terapêuticas e as novas tecnologias podem se tornar um fator estressante, passa a ser importante uma interface multimodal que possa monitorar o estado físico do jogador [Nery 2019]. Portanto, dispor de hardware apropriado a JS e que capture dados fisiológicos do autista é um recurso que auxiliaria tanto no ajuste do próprio jogo quanto no melhor entendimento da relação autista-jogo. Devido ao fato que a frequência cardíaca poder ser utilizada como um indício fisiológico do estresse do jogador [Loures 2002] e pelo fato de que muitos autistas apresentam sensibilidade sensorial atípica ao contato [Posar e Visconti 2018], este trabalho apresenta uma plataforma de JS capaz de verificar o batimento cardíaco do jogador de forma não invasiva, com intuito de utilizálo como parâmetro de ajuste do JS.

Este artigo apresenta na seção 2 trabalhos relacionados ao uso de biossinais em JS para TEA; na seção 3 está descrito o desenvolvimento de hardware da plataforma proposta; na seção 4 descreve-se o JS exemplo criado para testar a plataforma e, na seção 5, são apresentados e discutidos os dados das avaliações obtidas através de um questionário enviado a especialistas e interessados na área de TEA e JS. Por fim, na seção 6, apresentam-se conclusões e trabalhos futuros.

\section{Trabalhos Relacionados}

Em Fan et al (2018) foi classificado o estado emocional de indivíduos autistas durante treinamento de direção de automóveis, através da captura de dados fisiológicos de eletroencefalograma (EEG). Em Kuriakose e Lahirir (2017) foi desenvolvido um ambiente de realidade virtual (RV) para intervenções relacionadas com comunicação social para ser utilizado como ferramenta auxiliar em terapias para TEA. Enquanto o participante interage com o ambiente, a fotopletismografia de pulso (PPG), a atividade eletrodérmica (AED) e a temperatura da pele são capturados a fim de verificar o nível de ansiedade e fazer adaptações no ambiente para melhorar as habilidades de comunicação social do autista. Goulart et al (2014) apresentaram um sistema composto por um robô que cria atividades para autistas e que tem acoplado um sensor de EEG para caracterizar emoções do usuário.

Além de se notar que os sinais de EEG, PPG e AED são os mais comumente utilizados nos trabalhos estudados, foi observado que poucos se preocuparam em criar um aparato esteticamente atraente. Desta forma, as seguintes premissas foram escolhidas para o desenvolvimento deste estudo:

(a) captura da frequência cardíaca através de PPG, por se tratar de um método não invasivo;

(b) instalação de sensor de PPG de forma oculta no joystick;

(c) construção de um aparato com estética inspirada nos jogos de Arcade;

(d) elaboração de joystick atrativo ao jogador e;

(e) componentes de baixo custo financeiro. 
Isto gerou uma plataforma de hardware mostrada a seguir, que junto ao JS, foram submetidos à avaliação por especialistas.

\section{Apresentação da Plataforma}

\subsection{Fotopletismografia de Pulso}

PPG é uma técnica não invasiva que consegue detectar a variação de volume sanguíneo de um tecido através da verificação na mudança da absorção da luz emitida contra os vasos e capilares [Shelley e Shelley 2001]. O sensor de PPG utilizado neste trabalho é um sensor de baixo custo que apresenta um LED emissor de luz verde e um fotodiodo que recebe a reflexão desta luz. Ao posicionar o sensor de PPG em direção à pele, é possível detectar a frequência cardíaca, pois o aumento da pressão de pulso causará uma diferença mensurável na quantidade de luz refletida. O sensor PPG está conectado a uma placa eletrônica de prototipagem Arduíno Nano para fazer a conversão do sinal analógico para o sinal digital.

O diagrama da Figura 1 mostra todos os componentes eletrônicos utilizados na plataforma. O kit joystick padrão é composto por uma haste que possui 4 chaves do tipo fim de curso, que indicam para qual direção a haste é acionada.

As chaves da haste são conectadas na placa conhecida popularmente como Zero Delay (ZD), que tem como função converter os sinais do kit joystick para um padrão de comunicação, por exemplo USB. A placa ZD é conectada em uma das quatro portas USB disponíveis na placa Raspberry (RP) e a interface visual com o usuário é dada através de um monitor de 19" com alto-falantes embutidos e entrada HDMI para a conexão com a placa RP. Opcionalmente, foram utilizados teclado e mouse padrões nas entradas USB do RP durante a etapa de teste e programação, no entanto, posteriormente estes itens foram removidos, pois pode-se controlar o sistema remotamente via rede wireless. A empunhadura que acompanha o joystick padrão foi removida e em seu lugar foi acoplada a empunhadura com o sensor de PPG.

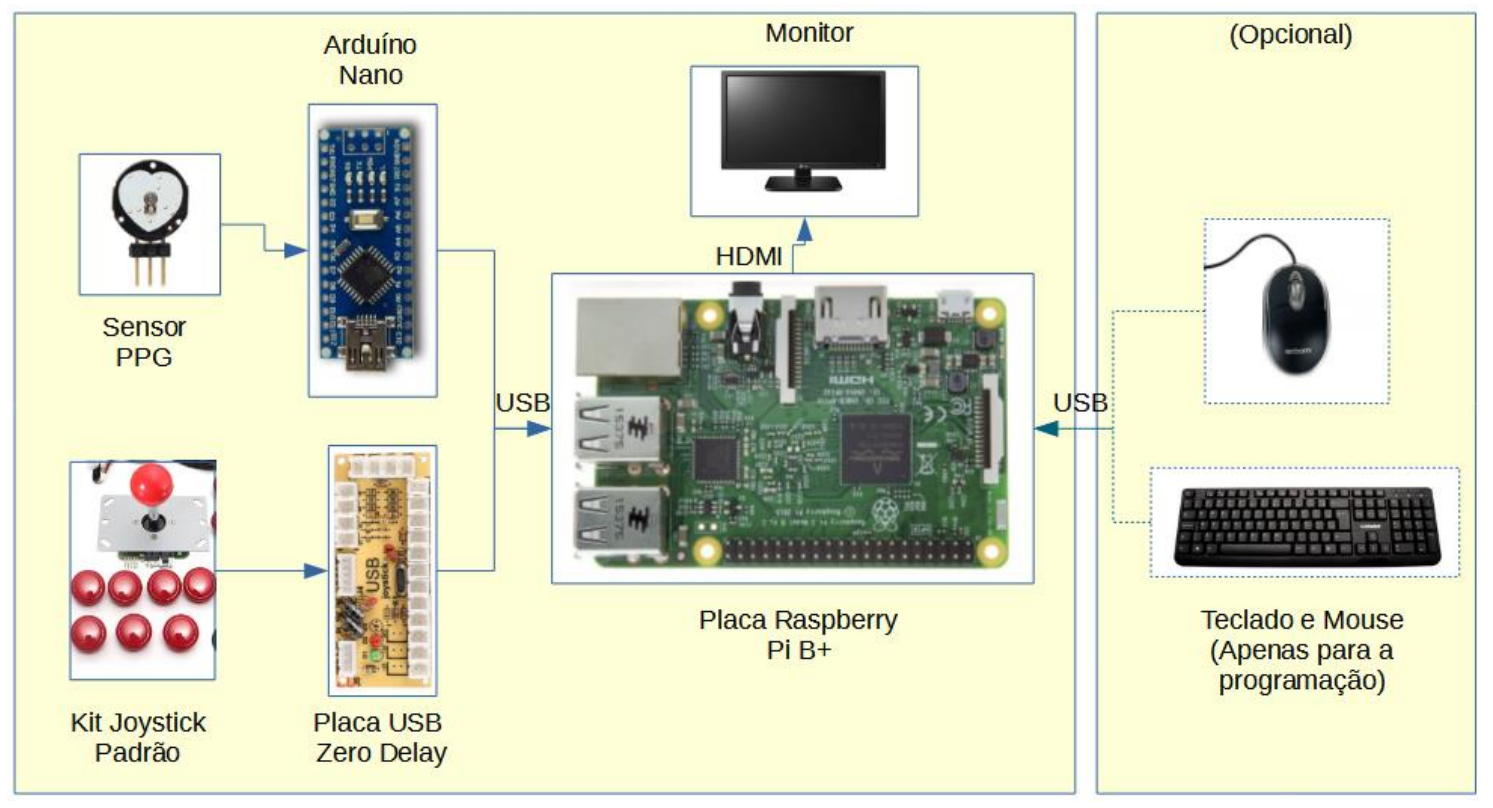

Figura 1. Diagrama de conexão dos componentes do sistema. 


\subsection{Empunhadura com Sensor PPG}

O modelo da empunhadura foi inspirado em uma pequena nave com um rosto caricato, a fim de ser mais atrativo para crianças, conforme mostra a Figura 2. Duas semiesferas compõem a empunhadura: a semiesfera inferior contém o encaixe para conexão com a alavanca do joystick padrão e a semiesfera superior aloja o sensor PPG, respeitando o espaçamento entre sensor-dedo através de um pequeno cilindro oco.

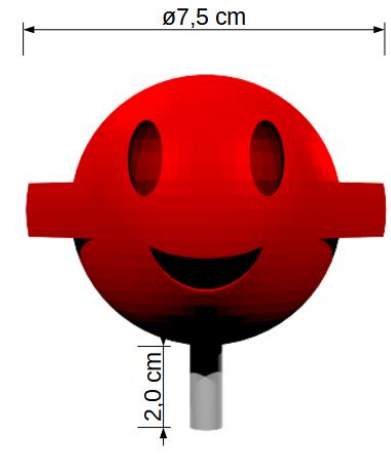

Vista Frontal

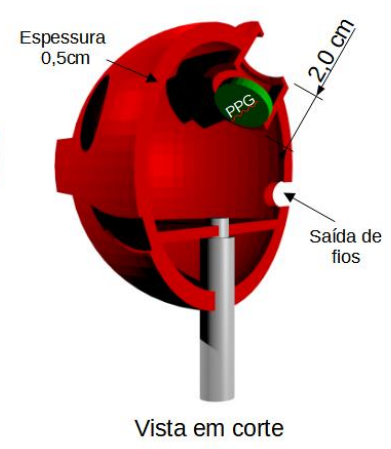

Vista em corte

Figura 2. Modelamento 3D da empunhadura em formato caricato.

O joystick foi centralizado no painel e 4 botões simetricamente opostos foram colocados a fim de atender jogadores destros e canhotos. Os botões também são diferenciados em tamanho para facilitar a seleção durante a interação com o JS como pode ser visto na Figura 3.

\subsection{Gabinete Arcade}

Para atender a premissa (c) do projeto, foi modelado um gabinete inspirado nos equipamentos Arcade com estilo "bartop", ou seja, é um gabinete projetado para ficar sobre alguma mobília, para flexibilizar a instalação do equipamento e atender crianças das mais diversas alturas e condições ergonômicas. Foi empregado madeira de $9 \mathrm{~mm}$ de espessura na construção do gabinete a qual foi pintada nas cores azul, amarelo, vermelho e verde, conforme mostra a Figura 3. Os alto-falantes do monitor estão direcionados para o jogador, contudo, o volume do som gerado pelo JS pode ser controlado, a fim de evitar distrações e desconfortos durante a intervenção. No interior do gabinete foi instalada a fonte de alimentação e as demais conexões elétricas.

\section{Desenvolvimento do JS Exemplo}

Para a verificação da funcionalidade da plataforma e da utilização do sinal de bpm como parâmetro de jogo, foi desenvolvido um JS exemplo com o objetivo de estimular a destreza óculo-manual, pois sabe-se que os déficits motores podem impactar na área cognitiva e social dos autistas [Ament 2015]. Foi escolhido o valor de $100 \mathrm{bpm}$ como ponto inicial para os testes da plataforma, pois o batimento cardíaco médio em repouso de crianças acima de 4 anos é menor que 100 bpm [Fleming 2011]. 


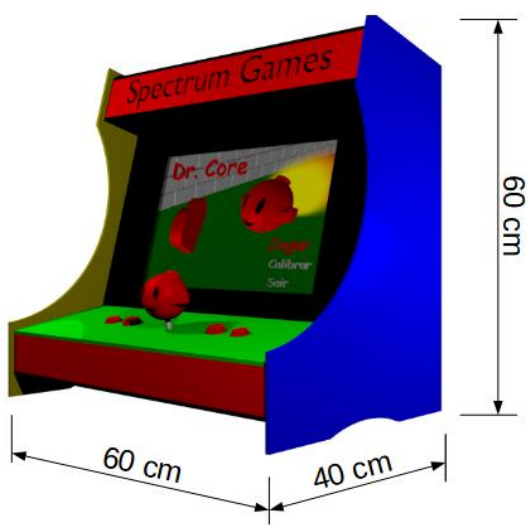

(a)

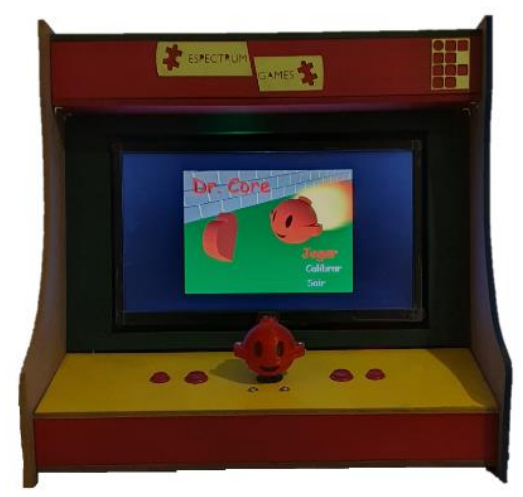

(b)

Figura 3. Em (a) modelo digital do gabinete e (b) vista frontal do gabinete fabricado em madeira.

\subsection{Interface de Calibração de BPM}

Ao selecionar a opção "Medir" na tela inicial, o jogador é direcionado para a interface de medição de bpm, após receber a instrução de como deve posicionar o dedo sobre o sensor, mostrada na Figura 4 (b).

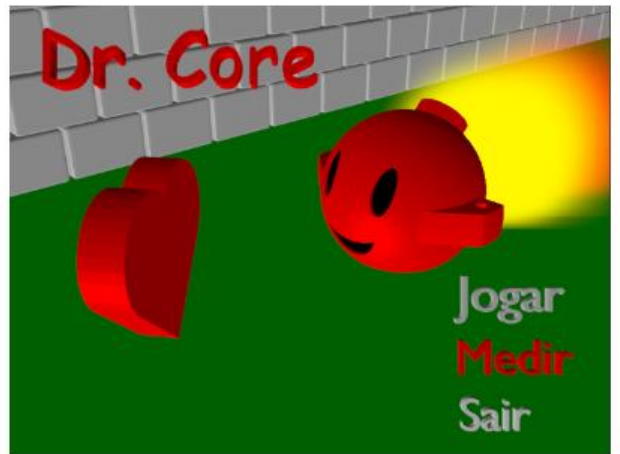

(a)

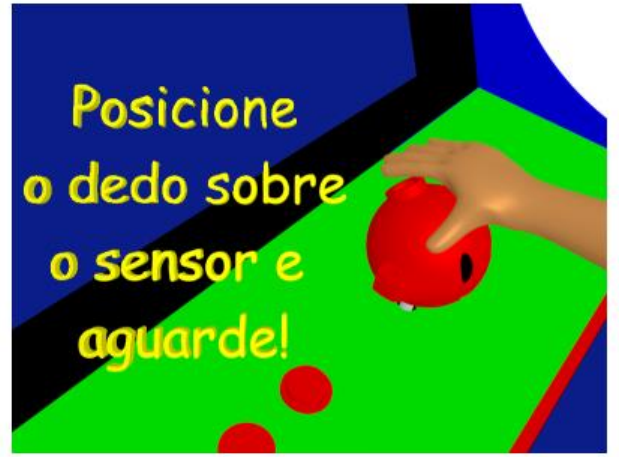

(b)

Figura 4. (a) Tela inicial do jogo “Dr. Core”, (b) Instrução de posicionamento do dedo para a medição de bpm utilizando o joystick.

\subsection{O Jogo "Dr. Core"}

Ao retornar para a tela inicial e selecionar a opção "Jogar", o jogador inicia a partida. O cenário é composto por uma vista superior de um castelo no qual o avatar, que tem o mesmo formato do joystick, é trancado e somente poderá sair ao término do tempo disponível.

O tempo é representado pelo escoamento de corações no interior de uma ampulheta, como pode ser observado na Figura 5. A velocidade de escoamento da ampulheta é dependente do valor de bpm medido pelo sistema na interface de calibração de bpm. Caso o valor seja maior ou igual a $100 \mathrm{bpm}$, o sistema aumenta em $50 \%$ o tempo original disponível para a captura de corações, com intuito de que o jogador possa diminuir o ritmo cardíaco e atingir o bpm médio em repouso. 
Os corações a serem coletados são gerados aleatoriamente no espaço interior delimitado pelas paredes do castelo. Para capturar um coração, o jogador deve, através da coordenação óculo-manual, direcionar o avatar até o coração, utilizando o joystick. $\mathrm{O}$ coração capturado desaparece da tela e o contador posicionado no canto superior esquerdo é incrementado, e caso ainda haja tempo disponível, um novo coração é posicionado aleatoriamente no cenário. Não há limites de corações por cenário, o único parâmetro limitador é o tempo disponível. Ao terminar o tempo disponível, o jogador é levado automaticamente para o próximo nível, em um total de 4 níveis.

A dificuldade de captura dos corações é aumentada através da inserção de barreiras no interior do castelo as quais o jogador deve desviar para alcançar os corações alvos, e, portanto, exigindo maior destreza do jogador. Após finalizar os 4 níveis, é possível verificar o desempenho do jogador através de um relatório disponibilizado na tela, que contém a quantidade de corações capturados, o tempo disponibilizado em cada nível e o valor de bpm utilizado no jogo. Este relatório pode ser registrado pelo terapeuta a fim de verificar a evolução do jogador no decorrer das seções. Após a apresentação do relatório, ao pressionar qualquer um dos botões, a tela de créditos é exibida e o jogador, ao pressionar qualquer botão novamente, é retornado à tela inicial, na qual tem a opção de jogar novamente, medir o bpm ou sair do jogo.

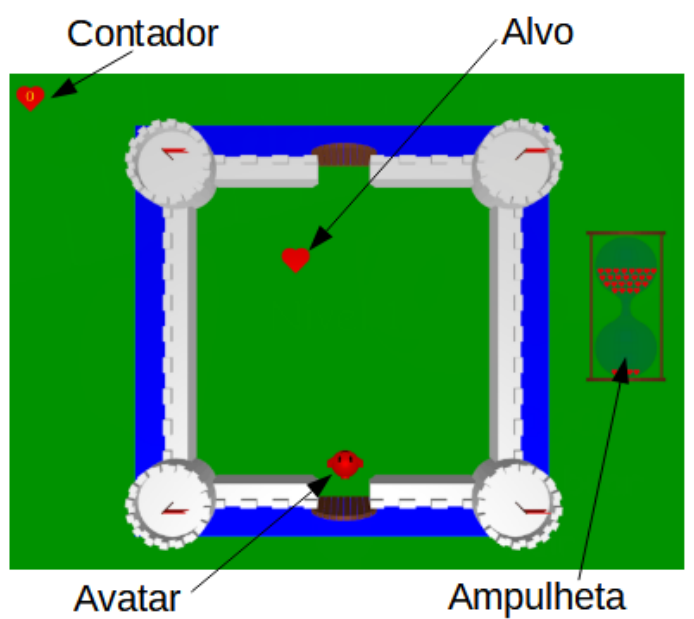

(a)

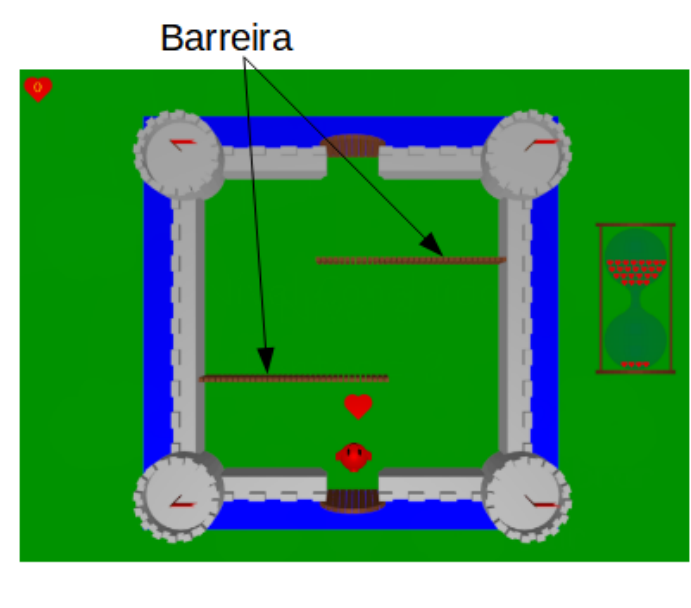

(b)

Figura 5. (a) Vista superior do Nível 1 e (b) Nível 4.

\section{Avaliação}

Abaixo apresenta-se a avaliação desta pesquisa, com foco na percepção de especialistas quanto ao potencial da plataforma arcade, do joystick e do jogo.

\subsection{Questionário}

Para verificar a opinião de especialistas e interessados na área de TEA e JS, foi criado um questionário digital contendo perguntas em relação à plataforma Arcade de JS proposta e sobre o jogo "Dr. Core", listadas na Tabela 1, o qual foi enviado à 258 pessoas que mostraram interesse em ambas as áreas por meio de participação em eventos promovidos pelo grupo de pesquisa e houve retorno de 39 participantes. 
As questões foram estruturadas em subgrupos de forma a obter a avaliação do entrevistado por assunto. Do item 1 ao 4, são informações pertinentes ao participante. $O$ gabinete em estilo arcade é avaliado do item 5 ao 8. A aparência do joystick e funcionalidade dos controles são apreciadas do item 9 ao 14. E do item 15 ao 18, estão as perguntas relacionadas ao JS exemplo. Para cada subgrupo, foi criado e disponibilizado no próprio questionário, um vídeo ilustrativo contendo as informações necessárias para que o avaliador pudesse opinar.

Tabela 1. Questionário de Avaliação.

\begin{tabular}{|c|c|}
\hline Item & Pergunta \\
\hline 1 & Em qual estado você reside atualmente? \\
\hline 2 & Qual sua área de atuação principal? \\
\hline 3 & Possui experiências que envolvam TEA? Caso sim, quanto tempo? \\
\hline 4 & Já teve ou tem contato com Jogos Sérios digitais? \\
\hline 5 & Custando $\$ 250$ dólares a plataforma é um recurso de baixo custo? \\
\hline 6 & A aparência da plataforma vai atrair a atenção dos autistas de 7 a 14 anos? \\
\hline 7 & $\begin{array}{l}\text { Plataforma Arcade é preferível para trabalhar com autistas do que um computador } \\
\text { convencional? }\end{array}$ \\
\hline 8 & $\begin{array}{l}\text { As dimensões de largura } \mathrm{x} \text { altura } \mathrm{x} \text { profundidade da plataforma são adequadas para } \\
\text { autistas de } 7 \text { a } 14 \text { anos? }\end{array}$ \\
\hline 9 & A aparência do joystick vai atrair a atenção dos autistas de 7 a 14 anos? \\
\hline 10 & Os controles são de tamanho adequado para autistas de 7 a 14 anos? \\
\hline 11 & $\begin{array}{l}\text { O sensor de batimentos cardíaco localizado no joystick não atrapalhará os autistas no uso } \\
\text { do jogo? }\end{array}$ \\
\hline 12 & Será fácil para os autistas entenderem o funcionamento dos controles? \\
\hline 13 & Será fácil os autistas lembrarem o funcionamento dos controles? \\
\hline 14 & Será intuitivo para os autistas usarem os controles? \\
\hline 15 & Conhecer o nível do batimento cardíaco (bpm) é importante para o controle do estresse? \\
\hline 16 & O uso do bpm como parâmetro de jogo está claro? \\
\hline 17 & O jogo Dr. Core ajuda a desenvolver a coordenação visual-motora? \\
\hline 18 & $\begin{array}{l}\text { O aumento do nível de dificuldade através das fases do jogo Dr. Core está coerente (Nível } \\
1 \text {-fácil, ..., Nível } 4 \text { - difícil)? }\end{array}$ \\
\hline
\end{tabular}

\subsection{Respondentes}

O principal local de residência dos participantes (item 1) é o estado de Santa Catarina (77\%), seguido de São Paulo (15\%), Minas Gerais (5\%) e Paraná $(3 \%)$. A área de atuação (item 2) de 41\% dos respondentes da pesquisa é a educação, $31 \%$ da tecnologia, 
$26 \%$ da área da saúde e $2 \%$ de outra área. Em termos de experiência com TEA e JS, verificou-se uma diversidade entre os participantes (ver Figura 6).

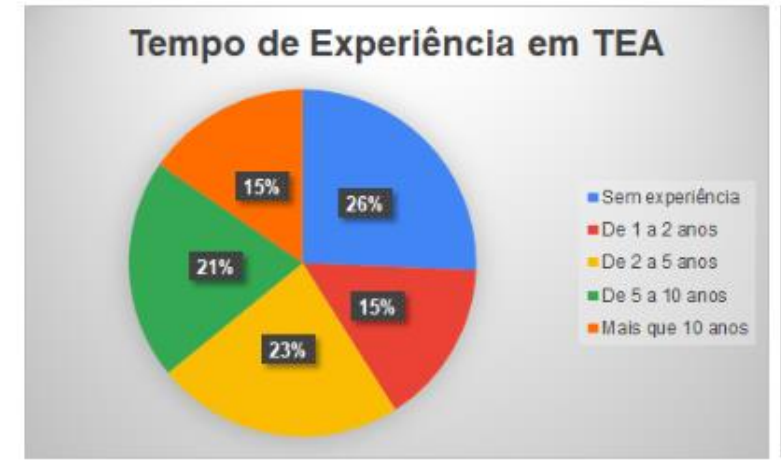

(a)

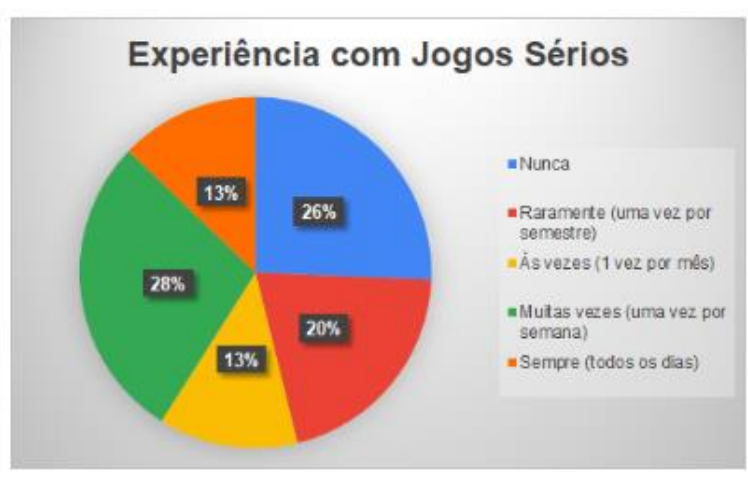

(b)

Figura 6. Tempo de experiência em TEA (a) e JS (b).

\subsection{Discussão}

Percebe-se, pela Tabela 2, que os entrevistados estão em concordância quanto à atratividade do gabinete e do joystick (itens 6 e 9) ao público autista, pois estas respostas apresentaram o menor desvio padrão entre respostas.

Tabela 2. Questionário de Avaliação, itens 5 ao 18.

\begin{tabular}{|c|c|c|c|c|c|c|c|c|}
\hline \multirow[b]{2}{*}{ Tema } & \multirow[b]{2}{*}{ Item } & \multicolumn{5}{|c|}{ Respostas (\%) } & \multirow[b]{2}{*}{ Média } & \multirow[b]{2}{*}{$\begin{array}{l}\text { Desvio } \\
\text { Padrão }\end{array}$} \\
\hline & & \begin{tabular}{|c|}
1 \\
Discordo \\
Totalmente \\
\end{tabular} & $\begin{array}{c}2 \\
\text { Discordo } \\
\end{array}$ & $\begin{array}{c}3 \\
\text { Neutro }\end{array}$ & $\begin{array}{c}4 \\
\text { Concordo }\end{array}$ & $\begin{array}{c}5 \\
\text { Concordo } \\
\text { Totalmente }\end{array}$ & & \\
\hline \multirow{4}{*}{ Arcade } & 5 & 10 & 38 & 31 & 13 & 8 & 2,7 & 1,066 \\
\hline & 6 & - & - & 18 & 64 & 18 & 4,0 & 0,599 \\
\hline & 7 & - & 11 & 32 & 43 & 14 & 3,6 & 0,853 \\
\hline & 8 & - & - & 38 & 49 & 13 & 3,7 & 0,669 \\
\hline \multirow{6}{*}{$\begin{array}{l}\text { Joystick e } \\
\text { Controles }\end{array}$} & 9 & - & - & 15 & 72 & 13 & 4,0 & 0,530 \\
\hline & 10 & - & 5 & 33 & 51 & 10 & 3,7 & 0,728 \\
\hline & 11 & 5 & 13 & 23 & 56 & 3 & 3,4 & 0,923 \\
\hline & 12 & 3 & 3 & 33 & 54 & 8 & 3,6 & 0,772 \\
\hline & 13 & - & 3 & 36 & 54 & 8 & 3,7 & 0,654 \\
\hline & 14 & 3 & 8 & 26 & 62 & 3 & 3,5 & 0,779 \\
\hline \multirow{4}{*}{ Jogo } & 15 & - & 3 & 5 & 54 & 38 & 4,3 & 0,677 \\
\hline & 16 & - & 8 & 5 & 62 & 26 & 4,1 & 0,783 \\
\hline & 17 & - & 8 & 8 & 51 & 33 & 4,1 & 0,841 \\
\hline & 18 & - & 3 & 10 & 64 & 23 & 4,1 & 0,656 \\
\hline
\end{tabular}

Conhecer o valor de bpm (item 15) também foi julgado como um importante fator para avaliar o nível de estresse, apresentando a maior média de avaliação. O que reitera a importância de plataforma multimodais como a apresentada neste trabalho. 
O custo de montagem (item 5) foi a característica da plataforma que obteve a maior discordância pelos participantes (maior desvio padrão) e a de menor concordância (de ser barata) por parte dos respondentes. Uma das possíveis causas é a alta variação da taxa cambial do Dólar do início do projeto comparada à taxa quando o questionário foi realizado. Outra possível causa é a área de atuação do respondente, pois, dependendo desta área, a percepção de custos de equipamentos pode variar.

Os entrevistados também se mostraram preocupados com o item 11, em relação à interferência do sensor na utilização do joystick pelo público autista, apresentando o segundo maior valor de desvio padrão e segunda menor média. Acredita-se que a possível causa deste resultado depende diretamente do tipo de experiência com pessoas autistas o respondente possui. Contudo, este item poderá ser melhor entendido quando uma avaliação prática com autistas for realizada.

A avaliação geral da plataforma (gabinete Arcade, controles e JS) pode-se dizer que foi favorável, pois excetuando o item 5, todos os outros itens questionados obtiveram nota média de avaliação acima do meio de escala (valor 3).

As medidas qualitativas de bpm realizadas com os autores do artigo mostraram que o sistema é capaz de entender a variação da frequência cardíaca, no entanto, esta medição de bpm não deve ser utilizada para fins de diagnósticos ou tratamentos relacionados à cardiologia, devido à suscetibilidade de variação de medição pela própria movimentação do dedo do jogador ao posicionar sobre o sensor ou outros fatores ambientais.

\subsubsection{Críticas e Sugestões}

Além das questões listadas na tabela 1, o questionário também oportunizava ao respondente fazer críticas e sugestões sobre a proposta da plataforma e do jogo Dr. Core. Em relação às vantagens e desvantagens que a plataforma arcade pode oferecer, destacamos as seguintes respostas: (a) "Melhor que o computador porque é direcionado. No computador muitas vezes a criança se frustra e quer mudar de atividade, dentro do próprio computador." (b) "Lembrando que cores fortes podem, ou não chamar a atenção de autistas. Há de se lembrar que TEA é um transtorno amplo e que abrange alunos de aprendizagens em níveis variados, e com funções diferenciadas." (c) "Para uma pessoa com Síndrome de Down será muito interativo e divertido, porém, com um autista, pode ocasionar fuga de demanda, pois o autista fica muito aficionado a uma coisa só." (d) "Tenho dúvidas de que um autista grave posicionará o indicador no sensor."

Já em relação às vantagens e desvantagens citadas para o jogo Dr. Core, ressaltamos as seguintes manifestações: (a) "Favorece movimentos de arcos da mão, que são importantes nas atividades do dia a dia." (b) "É legal para todas as crianças. Acho que idosos se beneficiariam também." (c) "Pode promover melhora na percepção de lateralidade, de discriminação de lados, na percepção e discriminação visual, no espectro de atenção, na resolução de problemas simples, no planejamento motor, na mobilidade da articulação do punho bem como na cinestesia (discriminar a incursão do movimento de um membro) deste membro, assim sendo, pode TALVEZ haver melhora para a integração e coordenação visual motora. Outro benefício que o jogo pode promover dependendo de como a criança/adolescente for abordada, é a aprendizagem de conceitos (em cima, embaixo, para frente, para o lado, dentro, fora, etc.) e quantidade $(1,2 \ldots)$. ." (d) "Poderia explorar melhor os elementos do jogo, o tamanho do avatar 
poderia ir diminuindo e o seu controle se tornando mais detalhado... isso sim testaria BEM a questão visual motora..., mas, não sei se o dispositivo tem sensibilidade suficiente para isso.... Em uma fase mais adiantada, os objetos poderiam se mover, o que tornaria o jogo mais interessante... Importante configurar as cores pois alguns autistas tem problemas com certas cores." (e) "Alguns indivíduos com autismo podem ficar ansiosos caso não consigam cumprir com o objetivo do jogo.”

\section{Conclusões e Trabalhos Futuros}

As premissas deste trabalho foram alcançadas com o desenvolvimento de um dispositivo capaz de medir qualitativamente o sinal de frequência cardíaca e utilizar esta informação como parâmetro de um JS. O apelo estético ao utilizar um gabinete nos moldes dos antigos jogos de entretenimento Arcade e a inserção do sensor de forma oculta em um joystick com forma agradável ao público autista também foram atingidos, como mostram os resultados do questionário feito com especialistas e interessados nos temas TEA e JS.

Uma plataforma para JS que se ajusta ao nível de estresse do jogador (pela captura da frequência cardíaca) pode ser uma valiosa ferramenta educacional tanto para pessoas como os autistas, os quais tem-se pouca informação sobre seus comportamentos durante o uso de jogos digitais, quanto para grupos neurotípicos com baixo nível de frustração, por exemplo (aspecto muito presente na população jovem da Geração Z).

Além da avaliação prática com indivíduos autistas, pretende-se futuramente aprimorar o trabalho através da verificação das condições ergonômicas do dispositivo em relação, por exemplo, a empunhadura do joystick por usuários destros e canhotos, posição de utilização (sentado ou em pé), distância ou tamanho de tela e amplitude sonora. Também será verificada a inserção de novos sensores não invasivos para sinais fisiológicos diversos, a fim de melhor entender o estado emocional e de estresse do usuário.

\section{Agradecimentos}

Os autores agradecem o apoio do IFSC Campus Joinville através do edital de pesquisa 02/DEPEC/CJ/2019. O terceiro autor agradece ao CNPq - Conselho Nacional de Desenvolvimento Científico e Tecnológico pela bolsa DT-II.

\section{Referências}

Alvarez, J. e Djaouti, D. (2011) "An introduction to Serious Game Definitions and Concepts", em Anais do Serious Games \& Simulation for Risks Management Workshop, pp. 11-15.

Ament, K. et al (2015). "Evidence for specificity of motor impairments in catching and balance in children with autism", J Autism Dev Disord., vol. 45, no. 3, pp. 742-51.

Fan, J. et al. (2018), "EEG-Based Affect and Workload Recognition in a Virtual Driving Environment for ASD Intervention", IEEE Transactions on Biomedical Engineering, vol. 65, no. 1, pp. 43-51.

Fleming, S. et al. (2011), "Normal ranges of heart rate and respiratory rate in children from birth to 18 years of age: a systematic review of observational studies", Lancet., vol. 377 , no. 9770 , pp. 1011-1018. 
Galvão, T. (2012) "Tecnologia Assistiva: favorecendo o desenvolvimento e a aprendizagem em contextos educacionais inclusivos", em C.R.M. Giroto R.B. Poker, S. Omote. (Org.). As tecnologias nas práticas pedagógicas inclusivas. Cultura Acadêmica, Marília-SP, pp. 65-92.

Goulart, C. et al. (2014), "EEG analysis and mobile robot as tools for emotion characterization in autism", em Anais do BMC 2014 8(Suppl 4): P85.

Kuriakose, S. e Lahirir, U. (2017), "Design of a Physiology-Sensitive VR-Based Social Communication Platform for Children with Autism", IEEE Transactions on Neural Systems and Rehabilitation Engineering, vol. 25, no. 8, pp. 1180-1191.

Loures, D. et al. (2002), "Estresse Mental e Sistema Cardiovascular", Arq. Bras. Cardiol., vol. 78, no. 5, pp. 525-530.

Néry, J. T. C. et al. (2019), "Jogos Sérios Multimodais para a Saúde: Um Mapeamento Sistemático da Literatura", em Anais do XVIII SBGames, pp. 198-206.

Oliveira, C. (2016) "Um Retrato do Autismo no Brasil”, Revista Espaço Aberto, Edição n. 170,6 de abril. São Paulo, Brasil.

Posar, A. e Visconti, P. (2018), "Sensory abnormalities in children with autism spectrum disorder”, J Pediatr (Rio J), vol. 94, no. 4, pp. 342-50.

Shelley, K. e Shelley, S. (2001), "Pulse Oximeter Waveform: Photoelectric Plethysmography, in Clinical Monitoring”, em Carol Lake, R. Hines, and C. Blitt, Eds.: W.B. Saunders Company, pp. 420-428.

Warren, Z. et al. (2011) "A Systematic Review of Early Intensive Intervention for Autism Spectrum Disorders”, Pediatrics, vol. 127, no. 5, pp. e1312 - e1322. 